Artigo de Revisão

\title{
Refletindo sobre o processo de institucionalização do idoso
}

\author{
Reflecting on the process of institutionalization of the elderly
}

\author{
Robson Rezende Dutra ${ }^{1}$, Sheila Cristina $\operatorname{Vargas}^{1}$, Luciana Tornquist $^{1}$, Debora Tornquist ${ }^{1}$, \\ Vanessa Amábile Martins ${ }^{1}$, Suzane Frantz Krug ${ }^{2}$, Valeriano Antonio Corbellini ${ }^{1}$ \\ ${ }^{1}$ Universidade de Santa Cruz do Sul, Santa Cruz do Sul, RS, Brasil. \\ .2 Pontifícia Universidade Católica do Rio Grande do Sul, Porto Alegre, RS, Brasil.
}

\begin{abstract}
RESUMO
Justificativa e Objetivo: O envelhecimento é um processo natural que vem obtendo maior relevância e preocupação a partir do aumento considerável da expectativa de vida da população. É observado que atualmente ocorre um aumento de estudos relacionados com o cuidado do idoso, em especial daquele que se encontra em uma Instituição de Longa Permanência (ILP). O objetivo deste artigo é discutir sobre o processo de institucionalização do idoso e sobre as características do grupo familiar e perfil da população internada nestas instituições. Conteúdo: O aumento da população idosa ao longo do tempo é acompanhado por modificações na estrutura e rotina familiar, fazendo com que, para muitas famílias, torne-se inviável atender as necessidades do idoso. Deste modo, as Instituições de Longa Permanência para Idosos (ILPIs) constituem-se em uma alternativa para suprir estas necessidades, proporcionando cuidado amplo pelo trabalho de equipe multidisciplinar. No entanto, deve-se ter uma atenção especial, para que não ocorra uma quebra no vínculo familiar do idoso, visto que a família é uma ferramenta essencial na qualificação do cuidado. Conclusão: É fundamental abandonar o conceito de que ao ingressar nas ILPIs o idoso está abandonado e desamparado, pois com a mudança na rotina familiar, a implantação da Política Nacional do Idoso e o aprimoramento das instituições, esta tornou-se uma alternativa importante para o bem-estar do idoso, permitindo acesso a uma assistência que, muitas vezes, não receberia em seu ambiente familiar, sendoimportante a manutenção do vínculo familiar, pois constitui-se em importante ferramenta para a qualidade de vida do idoso.
\end{abstract}

DESCRITORES: Saúde do Idoso. Idoso. Instituição de Longa Permanência para Idosos.

\section{ABSTRACT}

Background and objectives: Aging is a natural process that has achieved greater importance and concern from the considerable increase in life expectancy of the population. It is noted that currently there is an increase of studies related to elderly care, especially one who is in a long- 
stay institution (ILP). The aim of this paper is to discuss the elderly institutionalization process and the characteristics of the family group and profile of the hospitalized population in these institutions. Content: The increase in the elderly population over time is accompanied by changes in family structure and routine, so that, for many families, become unviable meet the needs of the elderly. Thus, the long-stay institutions for the Elderly (ILPIs) constitute an alternative to meet these needs, providing comprehensive care for multidisciplinary teamwork. However, one should pay special attention, so that there is a break in the family ties of the elderly, since the family is an essential tool in the care qualification. Conclusion: It is essential to abandon the concept that by joining the ILPIs the elderly are abandoned and helpless, because with the change in family routine, the implementation of the National Policy for the Elderly and the improvement of institutions, it has become an important alternative to the well-being of the elderly, allowing access to assistance that often do not receive in their home environment, it is important to maintain the family bond, as it constitutes an important tool for the quality of life of the elderly.

KEYWORDS: Health of the Elderly. Aged. Homes for the Aged.

\section{INTRODUÇÃO}

O aumento da população idosa é uma situação atual nos diversos países, sejam eles em desenvolvimento ou desenvolvidos. A diferença se dá pelo fato de que nos países desenvolvidos os fatores culturais, sociais e econômicos contribuem para aumento, ainda maior, da expectativa de vida em relação aos países em desenvolvimento. Este fenômeno ocorre no Brasil de uma forma acelerada, devido à diminuição dos índices da fecundidade e ao aumento considerável da expectativa de vida da população.

O acelerado processo de envelhecimento gera preocupações sociais e, principalmente, para a saúde, por atingir diretamente a capacidade funcional do indivíduo idoso. Além disso, acarreta algumas perdas que causam alterações na autonomia e independência do indivíduo, sendo a perda do papel social a mais significativa destas mudanças e que contribui para uma série de situações de exclusões do idoso. Em virtude destes fatores, o idoso procura espaços no qual possa se sentir aceito, seguro e cuidado, sendo uma alternativa a busca por Instituições de Longa Permanência para Idosos (ILPIs). ${ }^{2}$

Atualmente encontramos, tanto na literatura nacional, como na internacional, um aumento de estudos relacionados com o cuidado do idoso, em especial daqueles que se encontram em uma Instituição de Longa Permanência (ILP). Isto ocorre devido ao crescimento mais elevado da população idosa com relação aos demais grupos etários. ${ }^{3}$ Como consequência das mudanças na transição demográfica, houve um aumento dos índices de institucionalização dos idosos em diversos países, incluindo o Brasil, ainda que a atenção para a saúde deste grupo etário seja limitada. Esse aumento da busca por ILPI está transformando as instituições, vistas antes como símbolos de desfavorecimento e abandono, em opções de moradia para idosos com 
diferentes condições econômicas, requerendo das ILPIs ações de apoio social e de atenção à saúde do idoso. ${ }^{4}$

Diante deste contexto, o presente artigo tem como objetivo discutir sobre o processo de institucionalização do idoso e sobre as características do grupo familiar e perfil da população internada nestas instituições.

\section{MÉTODOS}

O estudo trata-se de uma revisão bibliográfica realizada com artigos publicados no período de 2008 a 2016, em periódicos nacionais e internacionais disponíveis nas bases de dados SciELO, LILACS e PubMed. Para esta busca, os descritores utilizados foram Saúde do Idoso; Idoso; Instituição de Longa Permanência para Idosos, sendo localizados no portal da Biblioteca Virtual em Saúde e na Biblioteca Eletrônica.

Após efetuar a procura nas bases de dados, procedeu-se a leitura dos resumos dos artigos para selecionar os textos que atendiam os critérios de acordo com as áreas temáticas abordadas e publicados nos idiomas inglês e português. Foram eliminados os artigos duplicados, os que não estavam de acordo com o tema proposto e os que não se apresentavam na integra nos sites da pesquisa. Foram selecionados 430 artigos nas bases de dados, onde 85 encontrados eram duplicados e 54 se enquadravam dentro dos descritores propostos. Destes, 20 atendiam o tema proposto no estudo.

Em seguida, as ideias e dados considerados mais relevantes foram agrupados e discutidos na construção de um referencial teórico.

\section{RESULTADOS E DISCUSSÃO}

Envelhecer é um processo que envolve diversidade, individualidade e variabilidade entre os indivíduos; ou seja, o processo de envelhecimento é uma experiência única para cada pessoa, diversificada entre membros de um mesmo grupo, e heterogênea, tanto individualmente, quanto em diferentes grupos sociais. ${ }^{5}$

Para Freitas, Queiroz e Sousa, o envelhecimento precisa ser percebido de forma integral e ser conservado como um processo biológico que apresenta efeitos psicológicos por expor alguns comportamentos característicos desta faixa etária. ${ }^{6}$ Assim sendo, o processo de envelhecer desfruta de uma crise existencial que transfigura a identidade da pessoa com o tempo, resultando em mudanças relacionadas ao mundo e sua história de vida. ${ }^{7}$ 
Simultaneamente com o vertiginoso aumento da população idosa, apareceram importantes transformações na composição e nos papeis da família, assim como nos modelos de trabalho e na migração, com jovens dirigindo-se para cidades a procura de trabalho, famílias com número reduzido de componentes, e a vitória das mulheres por um espaço no mercado de trabalho. Mudanças estas que por vezes acarretam na indisponibilidade das pessoas em consentirem as necessidades de cuidados estabelecidas pelo familiar que encontra-se na velhice. ${ }^{8}$

As ILPIs vem para suprir essas exigências, já que são destinadas a prover emas necessidades de moradia, higiene, alimentação e acompanhamento de saúde dos residentes. A Política Nacional do Idoso, em seu Decreto nº 1.948, de 1996, entende, por modalidade asilar, o atendimento em regime de internato, ao idoso sem vínculo familiar ou em que a família não tem condições de prover suas necessidades de moradia, alimentação, saúde e convivência social, garantindo assim o direito do idoso viver em uma dessas instituições. Além disso, devido à criação de legislações que envolvem os direitos dos idosos, as instituições vêm passando por um processo de transformação. ${ }^{9,10}$

Para Freitas e Noronha, as ILPIs são locais privilegiados, pelo fato de que se pode observar o modo de viver a velhice, caracterizando-se como um espaço repleto de conhecimentos e de diferentes histórias de vida. ${ }^{11}$ Mas, ao mesmo tempo em que é apontada por impressões positivas, também é marcada por impressões negativas sobre o sentido de ser idoso, passando, muitas vezes, a serem definidas como “depósito de idosos", onde a solidão, o desprezo e o abandono são os principais pensamentos que explicam a institucionalização de idosos que não possuem laços familiares ou de qualquer outra natureza.

Segundo Marin et al., o perfil dos idosos que residem em uma instituição se constitui em falta de um núcleo familiar, analfabetismo ou baixa escolaridade, pouca condição socioeconômica durante toda vida, trabalho infantil e a ausência de vínculos afetivos. ${ }^{12}$ Isto tudo geralmente transforma o idoso institucionalizado em uma pessoa sem interesse pela vida, sem expectativas e que demostra apenas a esperança de que um dia irá retornar ao ambiente familiar. Já para Freitas e Noronha, a visão do perfil do idoso residente na ILPI diz respeito ao indivíduo que embora possua família, opta por viver em uma instituição por causas variadas, como ser viúvo; não possuir filhos; escolher ser independente, ao invés de incomodar filhos e netos; dificuldade em contratar um bom serviço home care; temer maus-tratos familiares, entre outros. $^{11}$

Entretanto, a mudança radical de estilo de vida causada pela decisão de sair do seu lar para uma instituição, se configura para os idosos um grande desafio, pois se afastam de todo 
seu projeto existencial, enfrentando, muitas vezes, este processo como uma perda de liberdade, abandono dos filhos, fim da vida, além da ansiedade quanto aos cuidados realizados pelos funcionários. Porém, muitas vezes as instituições são vistas como um amparo para o idoso excluído da sociedade e da família, abandonado e sem lar fixo, transformando-se em um local que proporciona segurança, proteção e cuidado aos idosos, restabelecendo um pouco da alegria e contribuindo para uma nova inclusão social, ou seja, uma referência para uma vida e um envelhecimento digno. ${ }^{9,13}$

Mesmo que as instituições adquiram a responsabilidade do cuidado ao idoso quando este se encontra internado, ainda existe o inconveniente do afastamento do idoso com seu convívio familiar. Contudo, decidir por uma instituição de longa permanência não diz respeito a uma redução do valor da família para o apoio e cuidado dos seus membros dependentes, mas uma inovação na organização e na divisão de responsabilidades familiar. ${ }^{9,12,14}$

Por esta razão, proporcionar aos idosos resistentes um cuidado amplo, contemplando aspectos físicos, sociais, psíquicos, emocionais e espirituais, não diz respeito apenas à equipe multidisciplinar da instituição, que deve incentivar e manter os vínculos familiares dos idosos, mas também aos familiares deste, já que são ferramentas essenciais na qualificação do cuidado e da qualidade de vida do residente, caso contrário, pode caracterizar-se o abandono do mesmo. $^{15,16}$

De acordo com Santos et al., abandono é caracterizado quando a família não possui interesse no cuidado do idoso, diferentemente da família que não possui condições de cuidar. ${ }^{16}$ Ainda se considera que o abandono é motivado por situações referentes à fragilidade, ao término ou deficiência das relações afetivas e sociais da família com os idosos institucionalizados, ocasionando sentimentos diversos nos mesmos.

Devido a sua internação e o seu dia-a-dia na instituição, o idoso apresenta diferentes e conflitantes emoções, sendo o sentimento de exclusão, mágoa por ter sido abandonado e a crença de que é um estorvo para seus familiares, os mais encontrados, além dos de revolta e ingratidão, que estão ligados com sua história de vida no passado. Diante disso, aceitar a realidade se torna uma tarefa difícil durante a velhice, desafiando a instituição, que visa promover a saúde destes idosos, a estar preparada para proporcionar um acolhimento humanizado, facilitando assim a adaptação de seus residentes. ${ }^{9}$

Para Bessa e Silva, o processo adaptativo para o idoso institucionalizado é essencial, pois mesmo que alguns dos idosos tenham a liberdade de sair e de receber visitas, acabam algumas vezes ficando sem referências externas e, consequentemente, optando por não saírem, ou evitando visitas devido o constrangimento de ter, muitas vezes, hora marcada para entrada e 
saída. ${ }^{2}$ Desta forma, o sentido que muitos idosos dão sobre sua institucionalização não é apenas o de adaptar-se, mas resignar-se, submeter-se a esta situação por não possuir alternativa, existindo assim, um período de reorganização do seu cotidiano e da sua interação com os demais residentes.

Devido a esta importância da adaptação dos idosos, o Estatuto do Idoso estimula, em seu Art. 49, "a prestação de atendimento personalizado e a criação de pequenos grupos", pois essas atividades vêm como ferramentas para a melhora na relação entre os idosos residentes, além de ajudar a enfrentar o envelhecimento, pois a participação social, ou seja, a integração entre eles é um importante indicador de bem-estar para o idoso institucionalizado. Para isto, a preocupação por desenvolver atividades físicas para diminuir e prevenir distúrbios emocionais nos membros residentes está cada vez mais presente nas ILPIs. ${ }^{2,9,17}$

Cunha, Cunha e Barbosa afirmam que o apoio social auxilia na superação de determinados eventos na vida dos idosos, como a perda de alguém da família, da capacidade de trabalhar, perda de papéis sociais, o desejo de voltar para casa ou até mesmo a situação de internação na instituição. ${ }^{13}$ Desta forma, a rede de apoio e o convívio com outros residentes servem como manutenções da saúde das pessoas, contribuindo para um aumento da sobrevida e auxiliando os processos de cura.

Assim, o convívio de idosos em instituições de longa permanência converte-se em um contexto "familiar", no qual, os residentes encontram apoio nos próprios colegas, com trocas de experiências e histórias da mesma época, surgindo a partir disto, vínculos afetivos que se transformam em manifestações de carinho e confiança descobertos por meio deste convívio social nas instituições. Quebrando todos os estigmas e preconceitos referente as ILPIs que passam a ser a própria família dos idosos., ${ }^{2,9}$

Deste modo, as instituições de longa permanência não devem ser conceituadas somente como um local que ampara idosos largados ou abandonados pela família, mas também, devem ser abrangidas e respeitadas como uma opção de moradia no contexto da vida de cada pessoa e de cada grupo familiar, que procura a reorganização de sua vida. ${ }^{18}$ Miranda, Andrade e Furegato ressaltam que para compreender como vivem os idosos nas instituições, devemos primeiramente nos despir de qualquer preconceito, permitindo que o olhar escorra pelos espaços institucionais, com o intuito de perceber que estes ambientes não se caracterizam por locais inerentes, ociosos ou sem grandes acontecimentos. ${ }^{19}$ Residir em uma ILPI pode ser uma escolha para alguns dos idosos que não possuem condições de viver autonomamente, tornando-se verdadeiros lares que proporcionam apoio social, segurança, proteção, aconchego e cuidados de saúde que garantem uma qualidade de vida digna aos idosos. ${ }^{20}$ 


\section{CONCLUSÕES}

Por um longo período a institucionalização do idoso foi vista com negativismo, caracterizada pelo abandono do familiar e reservada aos marginalizados, desprovidos de um núcleo familiar e de laços afetivos. No entanto, é fundamental rever este conceito e abandonalo, pois, na atualidade, com a mudança na rotina familiar, em que muitas vezes os membros do grupo familiar encaram uma rotina corrida com diversas obrigações, e ainda com a implantação da Política Nacional do Idoso e o aprimoramento das ILPIs, a internação nestas instituições tornou-se uma alternativa válida e importante para o bem-estar e a qualidade de vida do idoso.

É importante superar a ideia de que ao ingressar nesta instituição o idoso está abandonado e desamparado e deixar de lado a culpabilização da família que opta por esta alternativa. Neste sentido, é essencial conhecer o trabalho realizado nas ILPIs, formadas por equipes multidisciplinares, que buscam além de suprir as necessidades do idoso, contemplar os diferentes aspectos (físico, psíquico, emocional, social) deste, permitindo acesso a uma assistência que, muitas vezes, o idoso em seu ambiente familiar não receberia. Cabe ressaltar que mesmo com a excelente assistência recebida nas ILPIs é importante a manutenção do vínculo com a família, pois esta constitui-se em importante ferramenta para a qualidade de vida do idoso.

\section{REFERÊNCIAS}

1. Soares LDA, Campos FACS, Araújo MGR, et al. Análise do desempenho motor associado ao estado nutricional de idosos cadastrados no Programa Saúde da Família, no município de Vitória de Santo Antão-PE. Ciênc saúde coletiva 2012; 17(5):12971304.http://dx.doi.org/10.1590/S1413-81232012000500023.

2.Bessa MEP, Silva MJ.Motivações para o ingresso dos idosos em instituições de longa permanência e processos adaptativos: um estudo de caso.Texto contexto- enferm 2008; 17(2): 258-65. http://dx.doi.org/10.1590/S0104-07072008000200006.

3. Gautério DP, Santos SSC, Pelzer MT, et al. The characterization of elderly medication users living in long-term care facilities. Rev esc enferm 2012; 46(6):1394-9. http://dx.doi.org/10.1590/S0080-62342012000600016.

4. Vitorino LM, Paskulin LMG, Vianna LAC. Quality of life among older adults resident in long-stay care facilities. Rev Latinoam Enfermagem 2012; 20(6):1186-1195. http://dx.doi.org/10.1590/S0104-11692012000600022.

5. Hajek A, Brettschneider C, Lange C, et al. Longitudinal predictors of institutionalization in old age. PloS ONE 2015;10(12).http://dx.doi.org/10.1371/journal.pone.0144203. 
6. Freitas MC, Queiroz TA, Sousa JAV. O significado da velhice e da experiência de envelhecer para os idosos. Rev esc enferm 2010;44(2): 407-412. http://dx.doi.org/10.1590/S0080$\underline{62342010000200024 .}$.

7. Mendes J, Soares VMN, Massi GAA. Speech therapy and nursing undregraduates perceptions on aging processes and education for elders care. Rev CEFAC 2015;17(2): 576585.http://dx.doi.org/10.1590/1982-021620153714.

8. Oliveira SC, Santos AA, Pavarini SC. The relationship between depressive symptoms and family functioning in institutionalized elderly. Rev esc enferm 2014; 48(1): 66-72. http://dx.doi.org/10.1590/S0080-623420140000100008.

9. Evangelista RA, Bueno AA, Castro PA, et al. Perceptions and experiences of elderly residents in asylum. Rev esc enferm 2014; 48(2): 85-91. http://dx.doi.org/10.1590/S0080$\underline{623420140000800013}$.

10. Gamburgo LJL, Monteiro MIB. Singularidades delenvejecimiento: reflexiones a partir de conversacionesconunanciano institucionalizado. Interface - Comunic, Saude, Educ 2009; 13(28): 31-41. http://dx.doi.org/10.1590/S1414-32832009000100004.

11. Freitas AVS, Noronha CV. Idosos em instituições de longa permanência: falando de cuidado. Interface - Comunic, Saude, Educ 2010; 14(33): 359-69. http://dx.doi.org/10.1590/S1414-32832010000200010.

12. Marin MJS, Miranda FA, Fabbri D, et al. Compreendendo a história de vida de idosos institucionalizados. Rev Bras Geriatr Gerontol 2012;15(1):147-154. http://dx.doi.org/10.1590/S1809-98232012000100016.

13. Cunha ANP, Cunha NNP, Barbosa MT. Geriatric teaching in Brazilian medical schools in 2013 and considerations regarding adjustment to demographic and epidemiological transition. Rev Ass Med Bras 2016; 62 (2): 179-183.http://dx.doi.org/10.1590/18069282.62.02.179.

14. Bessa MEP, Silva MJ, Borges CL, et al. Idosas residentes em instituições de longa permanência: uso dos espaços na construção do cotidiano. Acta paul enferm 2012; 25 (2): 177 82.http://dx.doi.org/10.1590/S0103-21002012000200004.

15. Michel T, Lenardt MH, Betiolli SE, et al. Meaning assigned by the elderly to the experience at a long stay institution: contributions for the nursing care. Texto \& contexto- enferm 2012;21 (3): 495-504.http://dx.doi.org/10.1590/S0104-07072012000300002.

16. Santos NO, Beuter M, Girardon-Perlini NMO, et al.The perceptions of workers in a longterm care institution for older adults regarding the family. Textocontexto- enferm 2014; 23 (4): 971-8.http://dx.doi.org/10.1590/0104-07072014003170013.

17. Santos FH, Andrade VM, Bueno OFA. Envelhecimento: um processo multifatorial. Psicol Estud 2009; 14 (1): 3-10. http://dx.doi.org/10.1590/S1413-73722009000100002. 
18. Almeida MFI, Barbosa AC, Lemes AG, et al. Depressão do idoso: o papel da assistência de enfermagem na recuperação dos pacientes depressivos. Rev Eletr Interdisc 2014; 1 (11): 107111.

19. Miranda FAN, Andrade OG, Furegato ARF, et al. Representação social da sexualidade entre idosos institucionalizados. JHSI 2015; 7 (1): 27-34. http://dx.doi.org/10.1590/0034$\underline{7167.2015680413 \mathrm{i} .}$

20. Oliveira JM, Rozendo CA. Long-stay institutions for the elderly: a place of care for those who have no choice? Rev Bras Enferm 2014; 67 (5): 773-79. http://dx.doi.org/10.1590/0034$\underline{7167.2014670515 .}$ 